\title{
Correlates of Physical Activity in a Cypriot Sample of Sixth-Grade Children
}

\author{
Constantinos A. Loucaides, Sue M. Chedzoy, \\ Neville Bennett, and Karen Walshe
}

\begin{abstract}
This study examined the association between self-reported physical activity and a number of potential correlates in a sample of 256 Grade 6 children. Physical activity was assessed in both summer and winter, and children and parents completed questionnaires assessing potential correlates of physical activity. Analyses revealed that gender, time spent playing outside, self-efficacy in overcoming barriers, and number of items of exercise equipment at home were variables associated with physical activity in both seasons. School location was a variable associated with physical activity only in the summer, whereas private-lesson attendance, sports-club attendance, and best friend's physical activity were variables associated with physical activity only in winter. Variance explained in physical activity were $42 \%$ and $51 \%$ in winter and summer, respectively.
\end{abstract}

There is now a substantial body of evidence indicating that physical activity accrues significant health benefits in adults, including a reduction in the incidence of cardiovascular disease and its risk factors (10). In childhood, the evidence relating physical activity to health is weaker (29), but recent studies utilizing objective measures of physical activity have documented a negative relationship between physical activity and overweight (16). This is of paramount importance, because children's weight has tended to increase in recent years (7). Nevertheless, there is increasing concern about children's low levels of physical activity $(3,20)$. Because the origin of many chronic diseases lies in early childhood (29), it is sound from a behavioral perspective to promote physical activity from the early years in order to reduce the incidence of lifestyle diseases in later life. In order to be effective, interventions aimed at promoting physical activity among young people must be based on knowledge about the determinants or correlates of participation in such activities (32). In this way, intervention programs might be more effective because they can target variables that are related to physical activity. A number of studies have been conducted with children that examined psychological, environmental, social, and demographic variables.

Loucaides is with Laniteio Elementary School, Limassol, Cyprus. Chedzoy, Bennett, and Walshe are with School of Education and Lifelong Learning, University of Exeter, Exeter, EX1 2LU, U.K. 
Physical activity is associated with a number of psychological variables that include perceptions of physical competence $(2,12)$, parents' perceptions of physical competence of their children (11), and enjoyment of physical activity (24). A self-efficacy scale consisting of three dimensions (support seeking, overcoming barriers, and competing activities) has also been used extensively (23). Evidence suggests that the overcoming-barriers construct of the self-efficacy scale is a consistent correlate of physical activity $(26,27)$. Environmental variables studied included time spent outside (1,21), television watching (14), access to exercise equipment at home (14,24), and sports-club attendance (27). Social variables associated with physical activity included parents transporting their children to places where they can be physically active (21) and physical activity of best friends and other family members (15). Regarding demographic variables, a recent review (20) classified gender as a consistent correlate of physical activity.

It is clear that physical activity is a multifactorial behavior influenced not only by personal and social factors but also by the environment. Therefore, in assessing determinants of physical activity one must examine variables from all potential categories. The multiple influences on physical activity have led researchers to apply ecological models to the study of physical activity. Based on these models, multiple levels of environmental influence can affect behavior (30). It is therefore crucial that variables proximal to the child, such as psychological, and distant from the child, such as the physical characteristics of the neighborhood, are investigated. An important consideration in the study of the correlates of children's physical activity is that $80 \%$ of the studies (20) were conducted in the United States. Therefore, the study of correlates in the Cypriot context might provide some areas for further investigation and also strengthen the existing body of knowledge. In addition, correlates of physical activity can differ depending on the variant climatic conditions during the year.

The purpose of this study was to assess potential correlates of physical activity in a sample of elementary school Cypriot children and to examine whether correlates of physical activity differ by season. Cyprus is an island situated in the northeastern part of the Mediterranean Sea. Access to the north part of the island is prohibited, because it has been occupied by Turkish troops since 1974. This study reports results from a larger study conducted on the free part of the island.

\section{Methods}

\section{Sample}

Participants for this study were 6th-grade students from five schools located in different geographical locations. Two of the schools were located in towns, one in Lefkosia, the capital of Cyprus, and the other one in Lemesos, a town located on the south coast. The other three schools were located in a coastal village, a mountain village, and an inland village. Consent forms were sent to all parents of Grade 6 children $(N=306)$. Signed consent forms were returned for 256 children $(83 \%)$.

\section{Measures}

Assessment of Physical Activity. A self-report instrument was developed from two validated self-reports, the Four by One-Day Recall $(4,5)$ and the Previous Day Physical Activity Questionnaire (PDPAR) (31). The choice of these 
two instruments was based on the facts that they correlated highly with objective measures of physical activity in validity studies and that both of these use a 1-day recall for assessing physical activity. Repeated 1-day recalls are more reliable than longer interval recalls (17). In addition, both instruments segment the day into time intervals in order to enhance the memory of the child. The Four by One-Day Recall $(4,5)$ protocol covered the school day of the child. The first question asked the child how he or she got from home to school and provided three options including car, bus, and walking. The second question asked whether the previous day had been a physical education day and provided a list of possible activities. Children had to indicate next to each activity the length of time they participated in that activity. The following questions asked the child to report the minutes that he or she engaged in activities during break times. For each break, a list of activities was provided. A space was also provided for "other activity." The duration of the physical education lesson and break time was written at the top of each question of the morning self-report, and children were instructed to think of that period only. Whereas the Four- by One-Day Recall is an interviewer-administered instrument, for the purpose of this study it was adapted as a self-administered instrument in order to reduce costs. In addition, perceived ratings of intensity were not used for scoring the physical activity self-report used in the present study, because previous studies indicated that children's ratings of intensity did not increase the validity of the instrument (22).

The PDPAR (31) protocol was adapted for the after-school part of the day. The after-school period was organized into fifteen 30-min blocks beginning at 1:30 p.m. and continuing to 9:00 p.m. A numbered list of 37 common activities was provided under three categories, activities with little or no movement, activities with a lot of movement, and transport. These categories were obtained from each school after focus-group discussions with the children. Whereas in the PDPAR protocol activities were grouped into seven categories, categories were reduced to three in the present study in order to make the instrument simpler for the children to complete, based on children's comments from a pilot study. In order to further enhance the children's memory, next to each 30-min block, each child was requested to write down the place where he or she was when engaging in that activity. Each student then entered the activity he or she participated in for the majority of each 30-min period.

The school part of the self-report was scored by multiplying the time spent on each activity by the assigned MET value (4). One MET is considered the resting metabolic rate during quiet sitting and is approximately equal to $1 \mathrm{kcal} \cdot \mathrm{kg}$. $\mathrm{hr}^{-1}(4,5)$. The after-school part of the self-report was scored by assigning a MET value to each 30 -min block and then multiplying the total time by its assigned MET value. The MET values from the school and the after-school self-reports were then added, and a total daily score was obtained. Only activities with a MET value of more than 4 were considered because this represents moderate-intensity activity, which is the target of the latest recommendations for children (6). Therefore, this self-report instrument provided an estimate of energy expenditure in kcal . $\mathrm{kg} \cdot \mathrm{day}^{-1}$ of moderate to vigorous physical activity (MVPA).

Pilot studies for this instrument were conducted in two phases. In the first phase, the self-report was given to a whole class of 6th-grade children to ensure that children understood the instructions and the layout of the instrument. In the second phase, the reliability of the scores of the instrument was established using 
one-way intraclass correlation coefficient. Test-retest reliability was .96 in a subsample of 40 children. Administration of the instrument took about $30 \mathrm{~min}$.

Correlates of Physical Activity. Two questionnaires were used to assess potential correlates of physical activity. One questionnaire was administered to children in order to assess psychosocial variables, and one questionnaire was administered to parents in order to assess social and environmental variables. The children's questionnaire consisted of items assessing self-efficacy (three subscales), based on the scale by Saunders et al. (23). Two further scales assessed children's perceptions of (a) parental beliefs about their children's physical competence, based on the scales used by Kimiecik et al. (12), and (b) parental encouragement of their children's physical activity. Two additional scales assessed children's enjoyment of physical activity and the frequency of their best friends' participation in physical activity in the after-school period. Responses to these scales were indicated via five-point Likert scales. A final scale assessed children's perceptions of physical competence, based on Harter's alternate-response-format scale (8). Items were scored on a four-point scale for this variable. Because lower scores on this scale represented higher perceptions of competence, scores were recoded.

Pilot studies with this instrument were also conducted in two phases. In the first phase, questionnaires were given to a whole class to make sure that children understood the items and the five-point-scale responses. The term physical activity was explained to the children by showing them six pictures with activities ranging from gardening and walking to sports. Items and response formats were revisited based on children's comments. In the second phase, questionnaires were administered to 46 children in order to establish the reliability of the instrument.

A second questionnaire was administered to children's parents. This questionnaire assessed potential environmental and social correlates of physical activity and consisted of items regarding daily time spent playing outside, watching television, and playing video games. In addition, the frequency per week that children attended sports clubs, attended sedentary private lessons, were transported by parents to places where they could engage in physical activity, and engaged in physical activity with their parents was assessed. Private lessons are lessons that children attend after school, such as English language or music. These items were scored on a six-point scale. Another item assessed the number of pieces of exercise equipment that children had available at home. Three additional items assessed the space available in the yard and the neighborhood and the safety of the neighborhood. In the final item parents indicated how their child traveled to and from school. Three options were provided: traveling by car, traveling by bus, and walking. These items were scored on a four-point scale.

\section{Procedures}

Self-reported physical activity was assessed for 4 weekdays in the winter (January and February) and for 4 weekdays in the summer (May and June). The researcher visited each school on a Monday, and children completed the questionnaire assessing potential psychosocial correlates of physical activity. This questionnaire was completed only in the winter, because changes in these psychosocial variables were not expected to take place during the short time interval between the two measurement waves. In addition, teachers expressed concerns about the time devoted to the research, and that children would miss out from their school subjects. 
Instructions were then given to classroom teachers on how to complete the physical activity self-report. Each self-report was completed at the beginning of the school day at 7:45 a.m. for the previous day. Children also took home questionnaires for their parents to complete during both summer and winter. Items assessing space available in the yard and neighborhood, safety in the neighborhood, number of items of exercise equipment at home, and sedentary-lesson attendance were not included in the summer questionnaire because changes in these variables were not expected to take place. This was based on information obtained from parents during a pilot phase of the questionnaire.

\section{Statistical Analysis}

Analyses were conducted with SPSS (version 9.0). Internal consistency reliabilities and 1-week test-retest reliabilities using one-way intraclass correlation coefficients were conducted for scales in the children's questionnaire assessing psychosocial correlates (see Table 1). Intraclass correlation coefficients were also computed for

\section{Table 1 Scales Used to Measure Potential Psychosocial Correlates of Children's Physical Activity and Reliability Coefficients}

\begin{tabular}{|c|c|c|c|c|}
\hline Scale & $\begin{array}{l}\text { Range } \\
\text { (number } \\
\text { of items) }\end{array}$ & Sample item & $\begin{array}{l}\text { Cronbach's } \\
\text { alpha }\end{array}$ & $\begin{array}{l}\text { Test- } \\
\text { retest } \\
\text { reliability }\end{array}$ \\
\hline \multirow{2}{*}{$\begin{array}{l}\text { Self-efficacy } \\
\text { support seeking }\end{array}$} & & & & \\
\hline & $1-5(8)$ & $\begin{array}{l}\text { How often do you think } \\
\text { you can ask your brothers } \\
\text { or sisters to do PA with you? }\end{array}$ & .79 & .86 \\
\hline $\begin{array}{l}\text { overcoming } \\
\text { barriers }\end{array}$ & $1-5(5)$ & $\begin{array}{l}\text { How often do you think you } \\
\text { can do PA even if it is hot } \\
\text { outside? }\end{array}$ & .81 & .81 \\
\hline $\begin{array}{l}\text { competing } \\
\text { activities }\end{array}$ & $1-5(6)$ & $\begin{array}{l}\text { I think I can do PA even if } \\
\text { I am in the house. }\end{array}$ & .67 & .82 \\
\hline Enjoyment of PA & $1-5(3)$ & I like doing PA. & .79 & .82 \\
\hline Best friends' PA & $1-5(2)$ & $\begin{array}{l}\text { How often do your best } \\
\text { friends engage in PA after } \\
\text { school? }\end{array}$ & .83 & .77 \\
\hline $\begin{array}{l}\text { Parents' } \\
\text { perceptions of PA } \\
\text { competence }\end{array}$ & $1-5(4)$ & $\begin{array}{l}\text { My mother or other adult } \\
\text { thinks I am good at PA. }\end{array}$ & .87 & .72 \\
\hline $\begin{array}{l}\text { Parental } \\
\text { encouragement } \\
\text { of PA }\end{array}$ & $1-5(3)$ & $\begin{array}{l}\text { My parents engage in PA } \\
\text { with me. }\end{array}$ & .64 & .64 \\
\hline $\begin{array}{l}\text { Perceptions of } \\
\text { competence }\end{array}$ & $1-4(7)$ & $\begin{array}{l}\text { I think I am good at PA/I } \\
\text { think I am not good at PA. }\end{array}$ & .79 & .75 \\
\hline
\end{tabular}

Note. $\mathrm{PA}=$ physical activity. 
the items on parents' questionnaires. These ranged from .79 to .99 and are reported elsewhere (13). Estimates of relative energy expenditure expressed in METs at an intensity $\geq 4$ METs representing MVPA derived over the 4 weekdays were added up and then averaged for summer and winter in order to obtain an activity score for each season.

Bivariate correlations were then computed between all potential correlates and physical activity scores separately for winter and summer. Significant correlations for each season were entered in hierarchical linear-regression models. Squared semipartial correlations were also computed. These indicate the unique contribution of each independent variable to the variability of the dependent variable (25). Variables were entered in the four blocks based on Welk's youth physical activity promotion model (30). Demographic variables, gender, and school location were entered in the first block because these are nonmodifiable variables, followed by psychological, social, and environmental variables. Because both distributions of physical activity were positively skewed, log transformations were applied in order to obtain normal distributions (25). All analyses were conducted with the transformed variables.

\section{Results}

Bivariate correlations between independent variables and MVPA are shown in Table 2. Hours per day playing outside had the highest correlation with MVPA in both winter and summer, with correlations of .40 and .45, respectively. Moderate correlations were also obtained for gender and self-efficacy in overcoming barriers in both seasons. Of interest is the negative correlation found between weekly frequency of private lessons in both summer and winter.

Results of the hierarchical multiple-regression analyses can be seen in Table 3. The variance explained in physical activity ranged from $42 \%$ in winter to $51 \%$ in the summer. All blocks of variables were significant in all analyses, with the exception of the social-variables block in the summer measurement. In winter, demographic variables explained $15 \%$ of the variance, with gender making a significant contribution in the final model. Psychological variables added an additional $9 \%$ to the variance, with self-efficacy in overcoming barriers contributing significantly to the model, and social variables contributed an additional $3 \%$ to the explained variance, with best friend's activity making a significant contribution. Finally, environmental variables accounted for an additional $15 \%$ of the variance in physical activity, with weekly club attendance, private lessons, exercise equipment at home, and hours spent playing outside contributing significantly to the final model. An examination of the squared semipartial correlations indicated that hours spent playing outside, gender, and private-lesson attendance had the highest unique contributions to physical activity variability.

In the summer, the demographic-variables block explained $31 \%$ of the variance in physical activity, with gender and school location making significant contributions in the final model. The block of psychological variables added $11 \%$ of the variance in physical activity, with self-efficacy in overcoming barriers being the sole significant contributor in this block. Finally, environmental variables contributed an additional $9 \%$ of the variance in physical activity, with exercise equipment at home and hours spent playing outside contributing significantly in the final model. An examination of the squared semipartial correlations showed that 


\section{Table 2 Bivariate Correlations Between Potential Correlates and MVPA in the Whole Group in Both Seasons}

\begin{tabular}{lcc} 
Variable & Winter & Summer \\
\hline Gender (boys = 1, girls = 2) & $-.38^{* * *}$ & $-.44^{* * *}$ \\
School location (urban = 1, rural = 2) & .09 & $.23^{* *}$ \\
SE in support seeking & $.27^{* * *}$ & $.21^{* *}$ \\
SE in overcoming barriers & $.38^{* * *}$ & $.39^{* * *}$ \\
SE in competing activities & $.20^{* *}$ & $.21^{* *}$ \\
Enjoyment of PA & $.23^{* * *}$ & $.21^{* *}$ \\
Best friends' PA & $.31^{* * *}$ & $.22^{* * *}$ \\
Parents' perceptions of PA competence & $.33^{* * *}$ & $.20^{* *}$ \\
Parental encouragement of PA & .12 & .13 \\
Perceptions of competence & $.27^{* * *}$ & $.29^{* * *}$ \\
Hours per day watching television & -.07 & -.09 \\
Hours per day playing video games & .07 & .05 \\
Hours per day playing outside & $.40^{* * *}$ & $.45^{* * *}$ \\
Weekly frequency of sports-club attendance & $.33^{* * *}$ & $.25^{* * *}$ \\
Weekly frequency of private lessons & $-.16^{*}$ & $-.24^{* * *}$ \\
Number of exercise equipment at home & $.25^{* * *}$ & $.18^{* *}$ \\
Weekly frequency parents transport child & $.25^{* * *}$ & .10 \\
Weekly frequency parents exercise with child & .10 & .02 \\
Space in yard & .09 & .08 \\
Space in neighborhood & .12 & .09 \\
Safety in neighborhood & .01 & .12 \\
Transport to school (by car or bus = 1, walk $=2$ ) & .10 & .11 \\
& & \\
\hline
\end{tabular}

Note MVPA = moderate to vigorous physical activity; $\mathrm{SE}=$ self-efficacy; $\mathrm{PA}=$ physical activity.

$* p<.05 . * * p<.01 . * * * p<.001$

gender, hours spent playing outside, and self-efficacy in overcoming barriers were the variables with the highest unique contributions to physical activity variability.

\section{Discussion}

Results from this study clearly indicate that physical activity is a multifactorial behavior (30), because significant associations were found among demographic, psychological, social, and environmental variables. Of particular importance is the similarity between results obtained in this study and those obtained in studies conducted in different populations. It is of interest that more variance in physical activity was explained by the summer measurement than by the winter measurement. Variables including sports-club attendance, private-lesson attendance, and activity of best friend were associated with physical activity at the multivariate 
Table 3 Results of Hierarchical Regression Analyses for Independent Variables and MVPA

\begin{tabular}{|c|c|c|c|c|c|c|}
\hline \multirow[b]{2}{*}{ Variable } & \multicolumn{3}{|c|}{ Winter } & \multicolumn{3}{|c|}{ Summer } \\
\hline & Partial $r$ & $\beta$ & $\begin{array}{c}R^{2} \\
\text { (adjusted) }\end{array}$ & $\begin{array}{c}\text { Partial } \\
r\end{array}$ & $\beta$ & $\begin{array}{c}R^{2} \\
\text { (adjusted) }\end{array}$ \\
\hline Demographics & & & $.147 * * *$ & & & $.307 * * *$ \\
\hline gender & $-.210 * * *$ & -.241 & & $-.252 * * *$ & -.301 & \\
\hline school location & & & & $.169 * *$ & .210 & \\
\hline Psychological & & & $.244 * * *$ & & & $.420 * * *$ \\
\hline $\begin{array}{l}\text { SE in overcom- } \\
\text { ing barriers }\end{array}$ & $.111 *$ & .163 & & $.211 * * *$ & .318 & \\
\hline Social & & & $.274 * *$ & & & N.S. \\
\hline best friend's PA & $.134 *$ & .154 & & & & \\
\hline Environmental & & & $.420 * * *$ & & & $.511 * * *$ \\
\hline $\begin{array}{l}\text { weekly club } \\
\text { attendance }\end{array}$ & $.155 * *$ & .233 & & & & \\
\hline private lessons & $-.200 * * *$ & -.213 & & & & \\
\hline $\begin{array}{l}\text { exercise } \\
\text { equipment }\end{array}$ & $.150 * *$ & .165 & & $.113^{*}$ & .123 & \\
\hline $\begin{array}{l}\text { hours playing } \\
\text { outside }\end{array}$ & $.248 * * *$ & .271 & & $.248 * * *$ & .286 & \\
\hline
\end{tabular}

Note . MVPA = moderate to vigorous physical activity; $\mathrm{SE}=$ self-efficacy $; \mathrm{PA}=$ physical activity; N.S. = not significant.

$* p<.05 . * * p<.01 . * * * p<.001$.

level only in winter, whereas school location was associated with physical activity only in the summer.

Sports-club attendance might be especially important in the winter, when the days are much shorter and the cold weather can restrict children's free play outdoors. This finding is in accordance with findings from the United States, where a number of studies indicated that participation in community sports and involvement in community-based physical activity organizations are significant correlates of physical activity in fifth- and sixth-grade children $(27,28)$. The unequivocal findings from two different cultures suggest that encouraging children's participation in sports clubs can increase their activity levels. Because the majority of sports clubs in Cyprus are privately owned, with parents having to pay fees and transport their children to the facilities, there is a need for community-based facilities that will allow access to a larger proportion of children at their locality. 
Of interest is the negative association of private-lesson attendance and physical activity in the winter months. This variable might be especially influential in winter because the days are shorter and, by the time children finish their lessons, there is no time left for free play. The emergence of this variable as a significant correlate of physical activity in the current study points to the fact that when one assesses determinants of physical activity, cultural trends should be taken into account. Physical activity of best friend was also found to be a significant correlate of physical activity in this sample during winter. Unfortunately, this variable was not assessed in the summer, and the lower correlation obtained during the summer might underestimate its true association with summer physical activity. Evidence from studies conducted in Hong Kong (9), the United States (18), and Estonia (15) also propose that promoting social interaction between children and their friends might help children increase their physical activity levels.

A number of variables including gender, self-efficacy in overcoming barriers, exercise equipment available at home, and time children spend playing outside contributed significantly to regression models in both summer and winter. The large variance contributed by the demographic-variables block, $15 \%$ and $31 \%$ in winter and summer, respectively, is worth noting. The negative correlation between physical activity and gender found in this study confirms previous research findings that girls' physical activity must be targeted for special intervention programs in order to increase their physical activity levels $(20,14,15)$. The significant contribution of school location in the summer measurement suggests that intervention programs to promote physical activity should also consider geographical location. Findings from this study suggest that children from town schools might be specially targeted for physical activity promotion in the summer months. Because there is inconsistent evidence regarding urban versus rural areas in physical activity levels (20), evidence from other cultural contexts is needed to support this finding.

In accordance with other studies that adapted a similar scale $(14,26,27,28)$, the present study revealed that self-efficacy was significantly associated with moderate to vigorous physical activity in both summer and winter. In multivariate analyses the overcoming-barriers construct was the only variable in the psychological block that was significant. To further explore the association of this variable with physical activity, bivariate correlations were computed between individual items and physical activity. In winter, the highest association was revealed with cold weather $(r=.36, p<.001)$ and in the summer with hot weather $(r=.35, p<.001)$. Children who were more confident in overcoming cold weather in the winter and hot weather in the summer engaged in higher activity levels. Because sheltered facilities for physical activity in schools and in public places are limited in Cyprus, constructing such facilities might increase children's confidence to be active in spite of weather conditions.

Time spent outside playing and exercise equipment available at home were two variables in the environmental block that were significantly related to physical activity in both seasons. Use of after-school time for playing outside was a variable significantly associated with physical activity in studies with 4-year-old children (1) and in a study with young people from Grades 4 through 12 (21). These consistent findings suggest that encouraging children to spend time outdoors playing might be one of the most effective ways to increase their physical activity levels. There is inconsistent evidence from studies in the United States 
regarding the association between exercise equipment available at home and physical activity participation. In one prospective study (27), this variable was found to be unrelated to moderate and vigorous physical activity, whereas two studies found significant associations between exercise equipment available at home and girls' physical activity $(24,28)$. This variable might be more important for girls' activity, because they might not be allowed to play outside as freely as boys.

Whereas the majority of the variables examined in this study were significantly related to physical activity at the bivariate level, few were significant at the multivariate level.

Any discussion of the results of this study would be incomplete without noting its drawbacks. The use of an unvalidated self-report to assess physical activity, as well as to assess independent variables, might have resulted in measurement error that suppressed associations (20). For example, studies have shown that self-reports of physical activity underestimate gender and ethnic differences in children's physical activity (19), and therefore data from self-reports should be treated with caution. In addition, the relatively small sample investigated did not allow gender-specific analyses to be conducted (25). Because gender is a consistent correlate of physical activity (20), gender-specific analyses might have illuminated the significant association of gender and physical activity observed in this study.

A number of advantages are also worth mentioning, however. The self-report instrument was designed from two validated instruments that have been used extensively in research studies. Furthermore, the satisfactory reliability coefficients found for the physical activity self-report, as well as for the scales assessing independent variables, increase our confidence in the quality of the measures. In addition, the assessment of physical activity over 4 days in both summer and winter and the examination of environmental and social determinants in both time periods are unique features of this study. Whereas the sample studied was not randomly selected, the schools chosen from diverse geographical areas of the island are perhaps indicative of the whole 6th-grade population of the island. Finally, the inclusion of variables from all domains, including demographic, psychological, social, and environmental, resulted in the models explaining $42-51 \%$ of the variance in physical activity.

In summary, the present study, conducted in a unique population, has largely confirmed results obtained from studies conducted in different populations. In this study, variables including gender, time spent playing outside, sports-club attendance, private-lesson attendance, self-efficacy in overcoming barriers, exercise equipment at home, and best friend's physical activity were significant correlates of physical activity. Furthermore, this study has suggested that different variables might be targeted at different times during the year and that urban school children might also be targeted for special interventions. Variables found to be consistently associated with physical activity in diverse samples of children should be targeted in intervention programs for promoting physical activity levels.

\section{Acknowledgments}

This article reports results from a study conducted for the fulfillment of the first author's PhD thesis at the University of Exeter under the guidance of Ms. Sue Chedzoy and 
Professor Neville Bennett. Special thanks are due to Professor Peter Preece for his help with statistical analyses.

\section{References}

1. Baranowski, T., W. O. Thompson, R. H. DuRant, J. Baranowski, and J. Puhl. Observations on physical activity in physical locations: age, gender, ethnicity, and month effects. Res. Q. Exerc. Sport 64:127-133, 1993.

2. Biddle, S., and M. Goudas. Analysis of children's physical activity and its association with adult encouragement and social cognitive variables. J. School Health 66:75-78, 1996.

3. Boreham, C., and C. Riddoch. The physical activity, fitness and health of children. $J$. Sport Sci. 19:915-929, 2001.

4. Cale, L. The Four by One-Day Recall Physical Activity Questionnaire: Protocol and Instruction Manual. Loughborough, UK: Loughborough University, 1993.

5. Cale, L. Self-report measures of children's physical activity: recommendations for future development and a new alternative measure. Health Educ. J. 53:439-453, 1994.

6. Cavill, N., S. Biddle, and J. F. Sallis. Health enhancing physical activity for young people: statement of the United Kingdom Expert Consensus Conference. Pediatr. Exerc. Sci. 13:12-25, 2001.

7. Freedman, D. S., S. R. Srinivasan, R. A. Valdez, D. F. Williamson, and G. S. Berenson. Secular increases in relative weight and adiposity among children over two decades: the Bogalusa Heart Study. Pediatrics 99:420-426, 1997.

8. Harter, S. The perceived competence scale for children. Child Dev. 53:87-97, 1982.

9. Johns, D. P., and A. S. Ha. Home and recess physical activity of Hong Kong children. Res. Q. Exerc. Sport 70:319-323, 1999.

10. Kesaniemi, Y. A., E. Danforth, M. D. Jensen, P. G. Kopelman, P. Lefebvre, and B. A. Reeder. Dose-response issues concerning physical activity and health: an evidencebased symposium. Med. Sci. Sports Exerc. 33:S351-S358, 2001.

11. Kimiecik, J. C., and T. S. Horn. Parental beliefs and children's moderate-to-vigorous physical activity. Res. Q. Exerc. Sport 69:163-175, 1998.

12. Kimiecik, J. C., T. S. Horn, and C. S. Shurin. Relationships among children's beliefs, perceptions of their parents' beliefs, and their moderate-to-vigorous physical activity. Res. Q. Exerc. Sport 67:324-336, 1996.

13. Loucaides, C. A., S. M. Chedzoy, and N. Bennett. Differences in physical activity levels between urban and rural school children in Cyprus. Health Educ. Res., Theory Pract. In press.

14. Pate, R. P., S. G. Trost, G. M. Felton, D. S. Ward, M. Dowda, and R. Saunders. Correlates of physical activity behaviour in rural youth. Res. Q. Exerc. Sport 68:241-248, 1997.

15. Raudsepp, L., and R. Viira. Sociocultural correlates of physical activity in adolescents. Pediatr. Exerc. Sci. 12:51-60, 2000.

16. Rowlands, A. V., R. G. Eston, and D. K. Ingledew. Relationship between activity levels, aerobic fitness, and body fat in 8- to10-yr-old children. J. Appl. Physiol. 86:14281435, 1999.

17. Sallis, J. F., S. A. Condon, K. J. Goggin, J. J. Roby, B. Kolody, and J. E. Alcaraz. The development of self-administered physical activity surveys for 4th grade students. Res. Q. Exerc. Sport 64:25-31, 1993. 
18. Sallis, J. F., T. L. McKenzie, J. P. Elder, S. L. Broyles, and P. R. Nader. Factors parents use in selecting play spaces for young children. Arch. Pediatr. Adolesc. Med. 151:414417, 1997.

19. Sallis, J. F., T. L. McKenzie, J. P. Elder, et al. Sex and ethnic differences in children's physical activity: discrepancies between self-report and objective measures. Pediatr. Exerc. Sci. 10:277-284, 1998.

20. Sallis, J. F, J. J. Prochaska, and W. C. Taylor. A review of correlates of physical activity of children and adolescents. Med. Sci. Sports Exerc. 32:963-975, 2000.

21. Sallis, J. F., J. J. Prochaska, W. C. Taylor, J. O. Hill, and J. C. Geraci. Correlates of physical activity in a national sample of girls and boys in grades 4 through 12. Health Psychol. 18:410-415, 1999.

22. Sallis, J. F., P. K. Strikmiller, D. W. Harsha, et al. Validation of interviewer-and selfadministered physical activity checklists for fifth grade children. Med. Sci. Sports Exerc. 28:840-851, 1996.

23. Saunders, R. P., R. P. Pate, G. Felton, et al. Development of questionnaires to measure psychosocial influences on children's physical activity. Prev. Med. 26:241-247, 1997.

24. Stucky-Ropp, R. C., and T. M. DiLorenzo. Determinants of exercise in children. Prev. Med. 22:880-889, 1993.

25. Tabachnick, B. G., and L. S. Fidell. Using Multivariate Statistics. New York: Harper Collins College, 1996.

26. Trost, S. G., R. P. Pate, M. Dowda, R. Saunders, D.S. Ward, and G. Felton. Gender differences in physical activity and determinants of physical activity in rural fifth grade children. J. School Health 66:145-150, 1996.

27. Trost, S. G., R. P. Pate, R. Saunders, D. S. Ward, M. Dowda, and G. Felton. A prospective study of the determinants of physical activity in rural fifth-grade children. Prev. Med. 26:257-263, 1997.

28. Trost, S. G., R. P. Pate, D. S. Ward, R. Saunders, and W. Riner. Correlates of objectively measured physical activity in preadolescent youth. Am. J. Prev. Med. 17:120126, 1999.

29. Twisk, J. W. R. Physical activity guidelines for children and adolescents. A critical review. Sports Med. 31:617-627, 2001.

30. Welk, G. J. The youth physical activity promotion model: a conceptual bridge between theory and practice. Quest 51:5-23, 1999.

31. Weston, A. T., R. Petosa, and R. P. Pate. Validation of an instrument for measurement of physical activity in youth. Med. Sci. Sports Exerc. 29:138-143, 1997.

32. Wold, B., and L. Hendry. Social and environmental factors associated with physical activity in young people. In: Young and Active? Young People and Health-enhancing Physical Activity: Evidence and Implications. S. Biddle, J. Sallis, and N. Cavill (Eds.). London: Health Education Authority, 1998, pp. 119-132. 